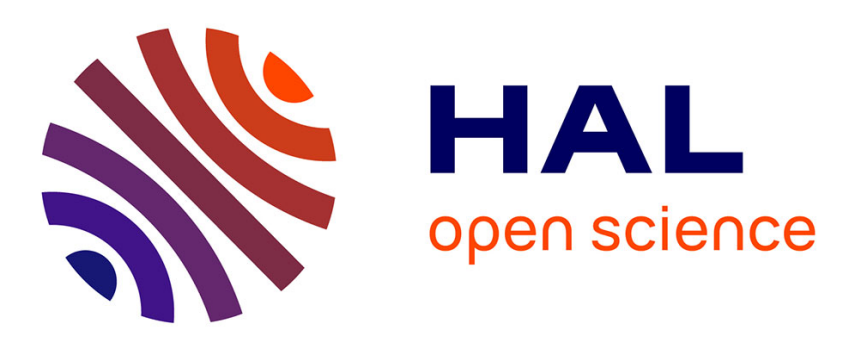

\title{
Vector Control of Piezoelectric Transducers and Ultrasonic Actuators
}

Sofiane Ghenna, Frédéric Giraud, Christophe Giraud-Audine, Michel Amberg

\section{To cite this version:}

Sofiane Ghenna, Frédéric Giraud, Christophe Giraud-Audine, Michel Amberg. Vector Control of Piezoelectric Transducers and Ultrasonic Actuators. IEEE Transactions on Industrial Electronics, 2018, 65 (6), pp.4880-4888. 10.1109/TIE.2017.2784350 . hal-01798120

\section{HAL Id: hal-01798120 \\ https://hal.science/hal-01798120}

Submitted on 23 May 2018

HAL is a multi-disciplinary open access archive for the deposit and dissemination of scientific research documents, whether they are published or not. The documents may come from teaching and research institutions in France or abroad, or from public or private research centers.
L'archive ouverte pluridisciplinaire HAL, est destinée au dépôt et à la diffusion de documents scientifiques de niveau recherche, publiés ou non, émanant des établissements d'enseignement et de recherche français ou étrangers, des laboratoires publics ou privés. 


\title{
Vector Control Of Piezoelectric Transducers and Ultrasonic Actuators
}

\author{
Sofiane Ghenna, Frédéric Giraud, Member, IEEE, Christophe Giraud-Audine, Member, IEEE, and \\ Michel Amberg
}

\begin{abstract}
This paper presents the implementation of a novel vibration amplitude control and resonant frequency tracking for piezoelectric transducers (PTs) and ultrasonic motors (USMs). It is based on a generalization of the vector control method to the PT and the USM, which is explained in the first part. We show that two independent controllers with a similar structure are required: one tracks the resonant frequency and the second controls the amplitude. We then present the implementation into a low-cost digital signal processing controller with a sampling period of $200 \mu \mathrm{s}$. Experimental results on a Langevin transducer achieved a time response of $20 \mathrm{~ms}$ approximately, and the generality of the method is further demonstrated on a 2-D tactile stimulator at the end of this paper.
\end{abstract}

Index Terms-Direct digital synthesis, energetic macroscopic representation (EMR), piezoelectric transducers (PTs), resonance tracking, ultrasonic transducer, vector control.

\section{INTRODUCTION}

Piezoelectric transducers (PT) are found in a growing range of applications, where a tool vibrating at high frequency is used. Examples include local heating as in ultrasonic welding [1] or surgery [2], generation of acoustic waves in a fluid as in cleaning [3] or acoustic levitation [4], ultrasonic motors [5], or active lubrication as in tactile feedback systems [6]. All these application requires large oscillations and therefore, in order to minimize the power consumption of the system, the PT should be operated at its resonance frequency, where the vibration to voltage amplitude ratio is higher. However, this optimal frequency strongly depends on several factors, e.g the dimensions of the transducer, the properties of the materials composing it, or the temperature. More importantly, the acoustic load coupled with the transducer can modify both the resonant frequency and the damping [7]. Finally, due to the non linear nature of the piezoelectric material, the resonance frequency may shift as the vibration amplitude increases [8]. Therefore, using PT often requires a control which acts both on the frequency and the amplitude of the excitation.

Since, for highly coupled PTs, the voltage and the current are virtually in phase at resonance [9], a tracking method consists in adjusting the excitation frequency until this condition is verified. This can be achieved with a Phase Locked Loop (PLL) [10] for example, and many authors have proposed different practical implementations of this principle [11],

The authors are with the Laboratoire d'Electrotechnique et d'Electronique de Puissance, EA 2697, HEl, Arts et Métiers ParisTech,École Centrale de Lille, Université de Lille, F-59000 Lille, France
[12]. However, these methods can not maintain a constant vibration amplitude when the load changes, and, since the voltage amplitude is not controlled, the power delivered to the transducer can not be increased in order to compensate the acoustical load impedance variation. To address this issue, some authors control the current amplitude at resonance [10], which is proportional to the vibration amplitude, by adjusting the voltage amplitude. This is done with a boost converter as in [2].

In general, the performances of these controls are measured only in steady state, i.e for constant or slowly varying reference values of the vibration setpoint. However, some applications require high dynamics, either to ensure a large bandwidth of the variation of the vibratory amplitude [13], or to reject perturbation induced by the high dynamic of the load [14]. In order to simultaneously address the resonant frequency tracking, and the high dynamic amplitude control, the concept of Vector Control Method (VCM) is generalized to piezoelectric actuators. The VCM has been successfully applied on a wide range of electromagnetic actuators [15] to reach outstanding performances in transitory operations or to realize sensorless drives. In the case of PT, the VCM allows both resonance fequency tracking and vibration amplitude control using two loops operating in parallel [16]. The VCM can also be used to synchronize ultrasonic transducers, in order to generate a traveling wave [17] for instance.

This paper presents the practical implementation of the VCM for PT, and typical performances obtained on experimental test bench, within the field of tactile stimulation although the exxtension to other application is straightforward. The paper is therefore organized as follows: we first present the modelling of a PT in a rotating reference frame, which is the core of the VCM. Then, we present the control in the rotating reference frame. We will show that the tracking of the resonant frequency and the amplitude control is deduced naturally by application of VCM. Its implementation on a DSP is then discussed and experimental results on a Langevin transducer are given. We will then conclude with the presentation of an application which requires the synchronization of several PTs to operate.

\section{Modeling in a Rotating Reference Frame}

\section{A. Energy Conversion in a PT}

A PT exploits the inverse piezoelectric effect to create internal stresses in the piezoelectric material when a voltage $v$ is applied. The stresses deforms the matter, generating and 
propagating a wave. A longitudinal wave is used in the case of a Langevin transducers, however transverse waves (Lamb or Rayleigh wave) can also be also used e.g in ultrasonic motors. Considering a specific point of the transducer, the vibration is only a function of time and denoted $w(t)$ hereafter.

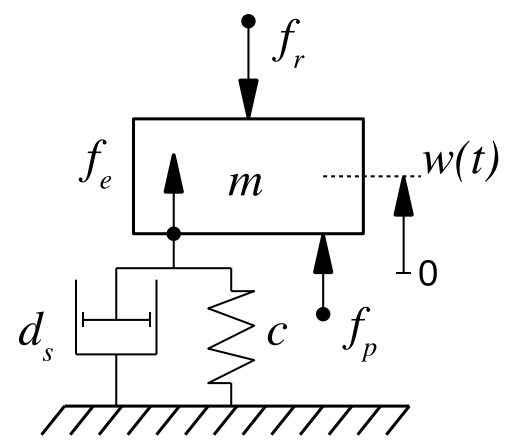

Fig. 1. Equivalent Mass-Spring-Damper model of the PT

A Spring-Mass-Damper Model is presented figure 1 which describes the behavior of PTs near resonance. In this model, the inverse piezoelectric effect produces a force named $f_{p}$ which is proportional to the voltage $v$, i.e $f_{p}=N v$ where $N$ is the conversion factor. Due to $f_{p}$ the mass $m$ is put in motion, while a visco elastic force $f_{e}=c w+d_{s} w$ counteract on it. The environment of the transducer is taken into account through the reaction force $f_{r}$. According to Newton's second law, one can write:

$$
m \ddot{w}+d_{s} \dot{w}+c w=f_{p}-f_{r}
$$

On the electrical side, a PT has a capacitive behavior, so the voltage $v$ is deduced from the current. In this paper, however, we assume that the voltage is perfectly controlled and imposed by the power supply. Hence, $v$ is an input of the system. Moreover, the vibration induces a motional current on the electrical part $i_{m}$, which is proportional to the vibration speed and we write:

$$
i_{m}=N \dot{w}
$$

note that conversion factor $N$ is the same for the piezoelectric force and the motional current, which results from the assumption that the conversion is lossless.

This model is useful for describing the instantaneous evolution of the vibration. However, in many applications, describing the amplitude is more of interest than the vibration itself. Therefore, we present the model in a rotating reference frame.

\section{B. Definition of the rotating reference frame}

Since PT are mechanical resonators, the vibration $w$ is a sinusoidal function of time, and one can use the complex notation $w(t)=\Re(\underline{w})$ with:

$$
\underline{w}=\underline{W}(t) e^{j \omega t}
$$

where $\underline{W}$ is the complex vibration amplitude which conveys simultaneously the amplitude of the vibration and its phase with respect to some arbitrary reference that will be defined later. $\Re($.$) denotes the real part and \omega$ is the pulsation of the vibration. This representation can be extended to the other variables, and we define $\underline{V}$ as a complex number relative to the voltage. Figure 2 shows the positions of the aforementioned complex variables at a given time $t$.

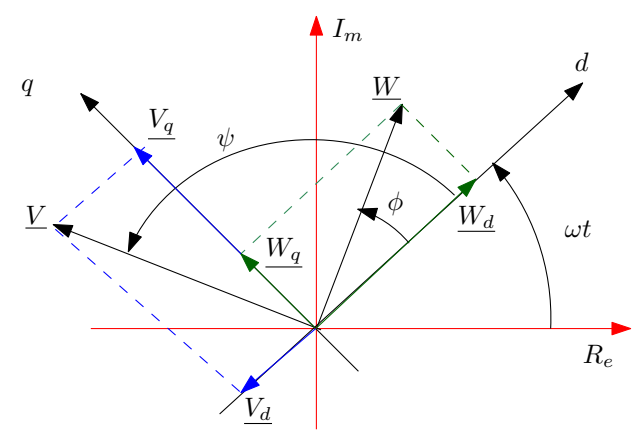

Fig. 2. Representation of the voltage and vibration amplitude in a rotating reference frame

During operation, the vectors affixed to the complex amplitudes are rotating. It is then convenient to project them in a rotating reference frame to obtain fixed vector in steady state. For that purpose, we denote:

$$
\begin{aligned}
\underline{w} & =\left(W_{d}+j W_{q}\right) e^{j \omega t} \\
\underline{v} & =\left(V_{d}+j V_{q}\right) e^{j \omega t} \\
\underline{f}_{r} & =\left(F_{r d}+j F_{r q}\right) e^{j \omega t}
\end{aligned}
$$

We define a rotating reference frame $(d, q)$, where the axis $d$ is by convention aligned with $e^{j \omega t}$. The objective of the VCM is then to give the evolution of the complex amplitudes, which is equivalent to the modeling in the rotating reference frame.

\section{Modeling in the rotating reference frame}

The equation 1 is supposed to be also valid for the complex vectors, and we write:

$$
m \underline{\ddot{w}}+d_{s} \underline{\dot{w}}+c \underline{w}=\underline{f}_{p}-\underline{f}_{r}
$$

The problem stated by equation (5) is general. However, to yield a less general, yet implementable model, we assume at this stage several simplifying hypotheses:

- The frequency is slowly varying, so the derivatives of $\omega$ are negligible,

- The dynamic of $\underline{W}$, that is the closed-loop bandwidth, is smaller than $\omega$.

From the equation (4), we can calculate the derivative of $\underline{w}$, which is simplified by neglecting $\dot{\omega}$ as stated in the first assumption:

$$
\underline{\dot{w}}=\left(\left(\dot{W}_{d}+j \dot{W}_{q}\right)+j \omega\left(W_{d}+j W_{q}\right)\right) e^{j \omega t}
$$

It is convenient to calculate the dynamic of the amplitude of the vibrating speed, rather than the amplitude of the vibration, hence, let:

$$
\underline{u}(t)=\underline{\dot{w}}=\left(U_{d}+j U_{q}\right) e^{j \omega t}
$$


leading to

$$
U_{d}=\dot{W}_{d}-\omega W_{q} \quad \text { and } \quad U_{q}=\dot{W}_{q}+\omega W_{d}
$$

and

$$
\ddot{w}=\left(\left(\dot{U}_{d}-\omega U_{q}\right)+j\left(\dot{U}_{q}+\omega U_{d}\right)\right) e^{j \omega t}
$$

Using the equations 8 , leads to

$$
W_{d}=\frac{U_{q}}{\omega}+\frac{\dot{U}_{d}}{\omega^{2}}-\frac{\ddot{W}_{d}}{\omega^{2}}
$$

Since the dynamic of $W_{d}$ is limited by $\omega$, it is reasonable to neglect $\frac{\ddot{W}_{d}}{\omega^{2}}$ compared to $W_{d}$ for a non-zero amplitude vibration. According to this assumption, and following a similar process with $W_{q}$, one can write:

$$
\underline{w}=\left(\left(\frac{U_{q}}{\omega}+\frac{\dot{U}_{d}}{\omega^{2}}\right)+j\left(-\frac{U_{d}}{\omega}+\frac{\dot{U}_{q}}{\omega^{2}}\right)\right) e^{j \omega t}
$$

Introducing (11), (9) and (7) into (5), and identifying the real and imaginary part, we obtain two new equations, describing the dynamic of the vibration speed in the rotating reference frame:

$$
\begin{aligned}
& \left.\left(m+\frac{c}{\omega^{2}}\right) \dot{U}_{d}+d_{s} U_{d}+\left(\frac{c}{\omega}-m \omega\right) U_{q}=F_{p d}-F_{k 12}\right) \\
& \left.\left(m+\frac{c}{\omega^{2}}\right) \dot{U}_{q}+d_{s} U_{q}-\left(\frac{c}{\omega}-m \omega\right) U_{d}=F_{p q}-F_{k q} 3\right)
\end{aligned}
$$

This model in a rotating reference frame shows that the forces in axis $d$ control the dynamic of the vibration velocity $U_{d}$, the environment acts as a perturbation (through $F_{r d}$ ) and there is a coupling between the axis. Same conclusion applies on axis $q$ which is controlled by $F_{q}$ with perturbation and coupling from the other axis.

PTs have high quality factor. It is, therefore, admitted that the pulsation of the vibration is close to the pulsation of resonance $\omega_{0}$. We name $\delta \omega$ the deviation of $\omega$ from $\omega_{0}$ such that $\omega=$ $\omega_{0}+\delta \omega$ with $\delta \omega \ll \omega_{0}$. We consider then that $m+\frac{c}{\omega^{2}} \simeq 2 m$ and $\frac{c}{\omega}-m \omega \simeq-2 m \delta \omega$. The equation 13 simplifies then to:

$$
\begin{aligned}
& 2 m \dot{U}_{d}+d_{s} U_{d}-2 m \cdot \delta \omega \cdot U_{q}=F_{p d}-F_{r d} \\
& 2 m \dot{U}_{q}+d_{s} U_{q}+2 m \cdot \delta \omega \cdot U_{d}=F_{p q}-F_{r q}
\end{aligned}
$$

We introduce two forces as follows:

$$
F_{d}=F_{p d}+2 m . \delta \omega \cdot U_{q} \quad \text { and } \quad F_{q}=F_{p q}-2 m . \delta \omega \cdot U_{d}
$$

The Energetic Macroscopic Representation (EMR, [18]), represents the system, highlighting the energy conversion (circles) and the energy storage (dashed rectangles) and therefore respects the causality. The EMR of a PT is given figure 3 in the rotating reference frame. It shows two coupled kinetic energy storage: one for the axis $d$ the other for the axis $q$. A coupling, which depends on the pulsation deviation with the resonance, shows that the two axis interact with each other. Such a coupling exists in electromagnetic machines, and the fact that this coupling depends on pulsation is a common feature that PTs share with them. This coupling is exploited in the next section, in order to implement original controls: control of the vibration speed, and tracking of the resonance frequency.

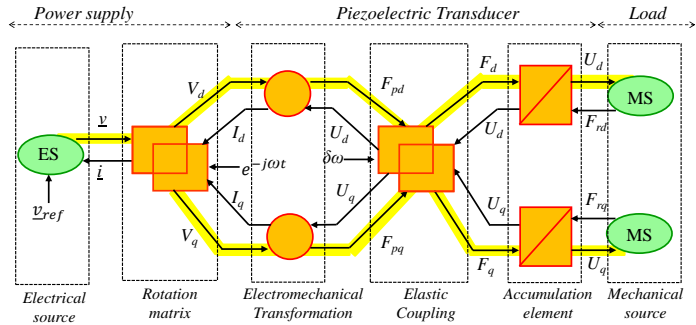

Fig. 3. EMR of the actuator in rotating frame

\section{Control of the vibration amplitude at constant pulsation}

In this section, the pulsation $\omega$ is fixed, and it is not necessarily the resonance frequency of the transducer. To fully control its vibration, the two outputs of the system namely $U_{d}$ and $U_{q}$ must be controlled. The step-by-step inversion of the EMR is given figure 4, and results in:

- two vibration controllers (which will be discussed later) represented as dashed blue boxes;

- the compensation of the couplings represented by the interlaced blue boxes;

- the inversion of the electromechanical coupling;

- the modulation at frequency $\omega$ realized by rotation of the vector $\underline{V}$ in order to obtain $v(t)$.

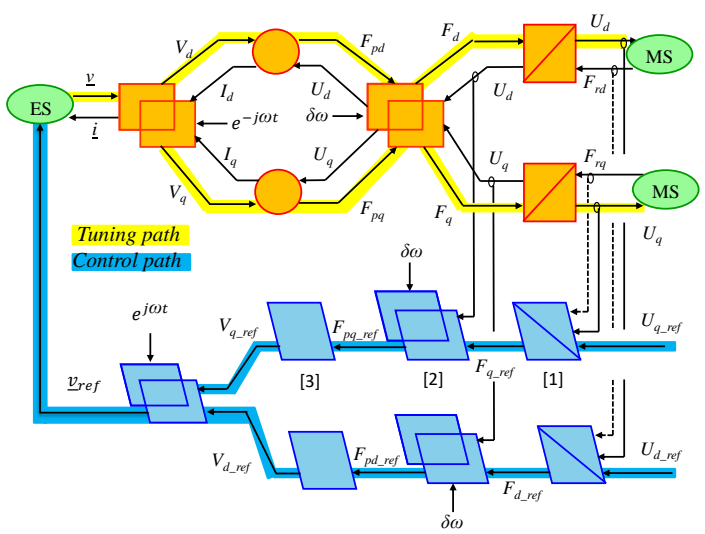

Fig. 4. EMR of the actuator in rotating frame and inversion-based control

Hence, $U_{d}$ and $U_{q}$ can be controlled by two independent loops in the rotating reference frame. These two loops can be implemented using similar structures. For instance, consider the control along the $q$ axis, represented by the block diagram depicted on figure 5 . The motor is represented by its transfer function which derives from the equation 15 :

$$
\frac{U_{q}}{F_{q}}=\frac{1}{d_{s}+2 m s}
$$

Since this transfer function is a first order, a simple PI is used to control the force $F_{q}$, and its parameters can be adjusted in order to obtain a predefined response time, without overshoot [19]. Moreover, since the dynamic in axis $d$ and $q$ are equal, the two PI controllers are the same. Finally, it is recommended to compensate for the effect of the external forces or the coupling in the other axis, but they can be omitted provided that the controllers are designed to reject these perturbations. 


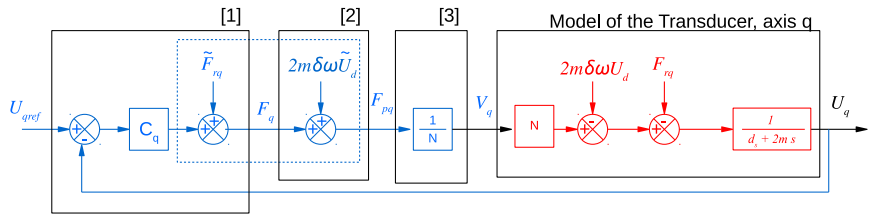

Fig. 5. Block diagram of the Control along the $q$-axis in the rotating reference frame; the labels refer to the block in the EMR of figure 4.

\section{E. Frequency tracking}

Considering $\delta \omega$ as an additional input of the system, the system has three inputs $\left(V_{d}, V_{q}\right.$ and $\left.\delta \omega\right)$ to achieve two criteria $\left(U_{d}=U_{d r e f}, U_{q}=U_{\text {qref }}\right)$ : the problem is underdetermined. Therefore, one input is arbitrarily set, and we choose to have let $V_{d}=0$. Moreover, it is generally admitted that for an efficient operation of the PT, it is necessary for the motional current to be in phase with the voltage. Since $V_{d}=0$, this leads to

$$
U_{\text {dref }}=0
$$

These aforementioned considerations lead to the EMR of the control depicted in figure 6; it shows two loops, operating in parallel:

- the vibration speed controller controls $V_{q}$ in order to obtain $U_{q}=U_{\text {qref }}$, and has been presented in the previous section

- the resonance tracking controller, which adjusts the value of $\delta \omega$ in order to have $U_{d}=0$

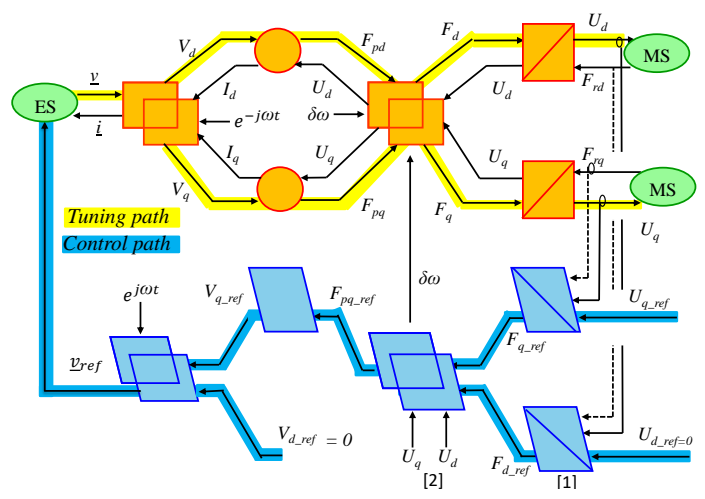

Fig. 6. EMR and the control for resonance frequency tracking

The implementation of the control loop of $U_{d}$ is presented figure 7. It looks very similar to that of Figure 5, and it also features an optional compensation of the force related to the environment $\left(F_{r d}\right.$ and $\left.F_{r q}\right)$. However, the inversion of the coupling yields a variable gain $\frac{1}{2 m U_{d}}$ in the block [2] of figure 7(as opposed to the constant gain $\frac{1}{N}$ in the previous controller). Hence, the frequency tracking controller can be the same as the velocity controller in axis $q$.

Unfortunately, this control is not suitable at low vibration amplitudei.e $U_{q}$ small. Indeed, inverting the relation 16 with small value of $U_{q}$ leads to large values of $\delta \omega$. To avoid this, the control is activated only when $U_{q}$ is above a threshold.

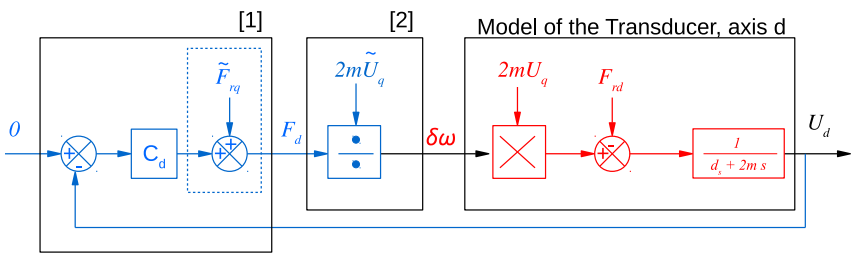

Fig. 7. Block diagram of the Control of the axis $d$ for the tracking of the resonance frequency; the labels refer to the block in the EMR of figure 6.

\section{EXPERIMENTAL RESULTS IN DIFFERENT EXAMPLES}

If the control of the piezoelectric transducers resembles that of the electromagnetic motors, its implementation is different because the pulsation of the supply voltage is much larger than the bandwidth of the vibration amplitude control. Moreover, the measured variables represent only the real parts of the corresponding complex vectors in the fixed frame (i.e the projections on the real axis on fig 2). It is therefore necessary to estimate in the vector in the rotating frame $\{d, q\}$. This implies to transport the real measurement in the rotating frame and reconstruct the missing imaginary parts of the various vectors. The practical implementation on a low cost DSP (STMicroelectronics STM32F4) is discussed below, as well as two experimental results on two applications, one requiring a resonance frequency tracking, the second operating at constant frequency.

\section{A. Practical implementation of the VCM}

The control scheme proposed in this paper involves two processes working at different sampling frequencies. A high speed process $\left(T_{e}=1 \mu s\right)$ that handles the $\{d, q\}$ transformation and is driven by a Digital Direct Synthesis (DDS) as shown in the dashed box of figure 8. The VCM, that defines the voltages and the frequency, and is updated at a slower frequency $\left(T_{s}=200 \mu s\right)$.

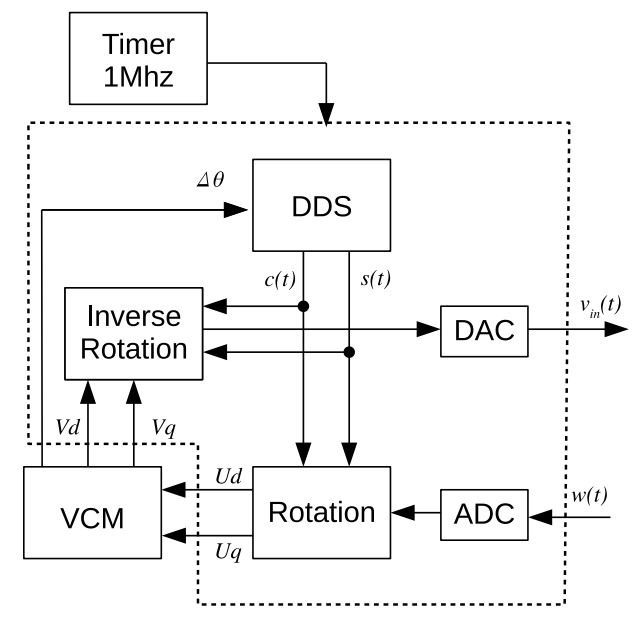

Fig. 8. Real-Time implementation of the VCM in a STM32F4.

Direct Digital Synthesis (DDS): The DDS produces the two sampled signals of $\cos (\omega t)$ and $\sin (\omega t)$, named $c$ and $s$ respectively. It consists in a $N=32$ bit accumulator named 
$R_{D D S}$. At each rising edge of a $1 \mathrm{Mhz}$ timer (providing a signal with a period $T_{e}=1 \mu s$ ), the register is increased by a quantity named $\Delta \theta$. Therefore, the DDS needs $T=\frac{2^{32}}{\Delta \theta} T_{e}$ to overflow, which is also the period of the voltage $v(t)$. The only 9 highest bits of $R_{D D S}$ are used as an input of a read-out table, where the values of cos and sin are stored in a memory coded on two 8 bits numbers, ranging from -128 to +127 .

These settings of the DDS allows us to fine tune the frequency of the voltage, with a resolution of $\frac{1}{2^{32} T_{e}}=0.2 \mathrm{mHz}$, which is enough for this application.

Inverse rotation: According to (4), one can write

$$
v(t)=V_{d} \cos (\omega t)-V_{q} \sin (\omega t)
$$

Hence, the Inverse Rotation consists in 2 multiplications and one addition. The values of $V_{d}$ and $V_{q}$ are given with signed 8 bits numbers. Therefore, the result is truncated to the 12 most significant bits, and sent to the Digital to Analog Converter Register.

Figure 9 shows the output voltage of the DSP when $V_{d}=0$ with $\Delta \theta=121762322$ leading to $\omega=2 \pi \times 28350 \mathrm{~Hz}$. The waveform evolves by steps as a result of discretization and digitalization.

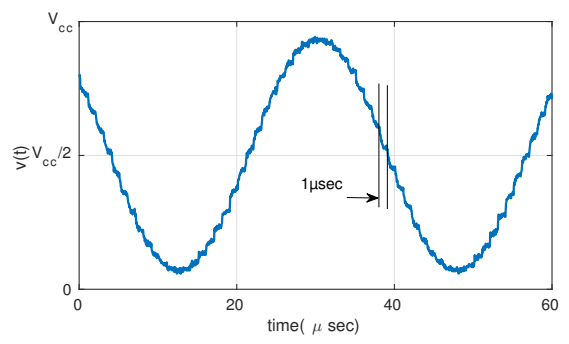

Fig. 9. Output voltage of the DSP; each step is $T_{s}=1 \mu \mathrm{sec}$ long.

Rotation: The rotation block computes the values $U_{d}$ and $U_{q}$ from the measurement $u(t)$, and we propose to use a Discrete Fast Fourier Transform. Using (4), one can write

$$
u(t)=U_{d} \cos (\omega t)-U_{q} \sin (\omega t)
$$

Considering that

$$
\begin{aligned}
& \frac{1}{T} \int_{0}^{T} u(t) \cos (\omega t) \mathrm{d} t=\frac{1}{2} U_{d} \\
& \frac{1}{T} \int_{0}^{T} u(t) \sin (\omega t) \mathrm{d} t=-\frac{1}{2} U_{q}
\end{aligned}
$$

The principle of the Rotation block is illustrated in figure 10. It consists in sampling $u(t)$ at each step $k T_{e}$ and multiplying this value by the signals $c$ and $s$ obtained from the DDS block. Then, the two resulting values are stored in 2 rotating buffers with equal length denoted by $N_{b}$. At the beginning of a period of the VCM $n T_{s}$, the $N_{b}$ elements of each buffer are summed in order to calculate the discrete integral of (21) and (22) and consequently $U_{d}$ and $U_{q}$.

The length $N_{b}$ of the buffers must be chosen so that it contains a number of samples spanning a duration as close as possible to an integer number of period, that is:

$$
N_{b}=E\left(\frac{T_{s}}{T} E\left(\frac{T}{T_{e}}\right)\right)
$$

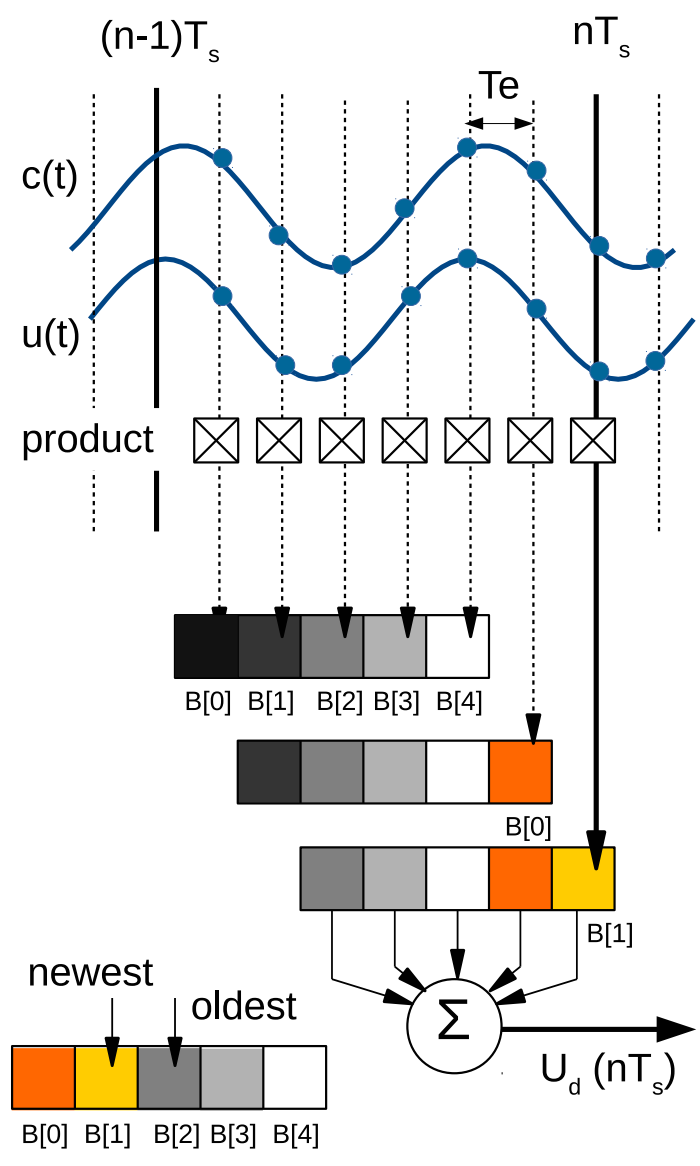

Fig. 10. Principle of the inversion block for $U_{d}$, with $T_{s}=7 T_{e}, T<5 T_{e}$ and $N_{b}=5$; $\bullet$ are sampled values of $c(t)$ and $u(t)$. The rotating buffer is named $\mathrm{B}$.

Where $E(x)$ is the integer part of $x$. Because the calculation of $U_{d}$ and $U_{q}$ is done on a large number of samples, this method is robust in the presence of noise and harmonic in the signal, and was found to give satisfactory results.

\section{B. Vibration amplitude and frequency tracking of a Langevin Transducer with VCM}

The proposed Vector Control Method (VCM) is illustrated for a Langevin transducer (FBL28252SSF-FC, Fuji Ceramics Corp, Japan) depicted in figure (11).

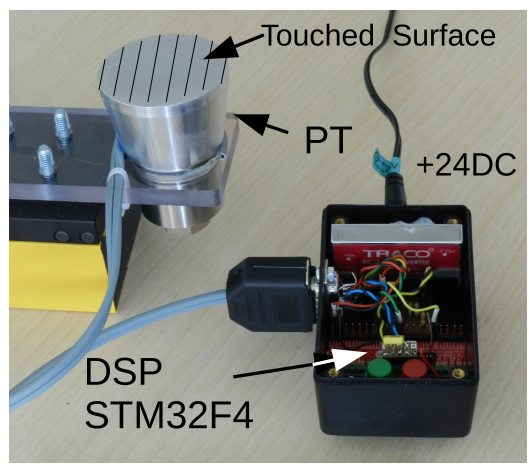

Fig. 11. The Langevin Transducer and its control and power electronics 
This transducer is used in an experiment on diminished haptics [20]. This involves vibrating a textured surface to several tens of kilohertz at about $2 \mu \mathrm{m}$ of amplitude. When the finger touches the vibrating surface, it is felt less rough than without vibration.

It is essential that the actuator always operates at the resonant frequency to ensure the lowest supply voltage and the best system efficiency. Indeed, to minimize the bulk of the power supply, it has little power reserve. These consideration allow us to use a linear amplifier (LM675 - Texas Instrument) mounted in non-inverting amplifier with a gain of 9, as presented figure 12. Because a DSP is used to supply the reference voltage to the amplifier, an input capacitor $C_{e}$ anf the resistor $R_{e}$ are added in order to cancel its mean value. Moreover, $R_{s}$ and $C_{s}$ are added to stabilize the amplifier.

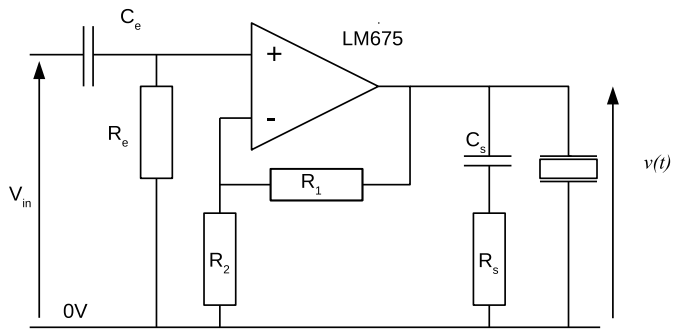

Fig. 12. The linear Amplifier.

The vibration velocity can be measured indirectly by measuring the current supplied to the to the piezoactuators if the latest is highly coupled and operating near the resonance frequency. Nonetheless, in this study, we preferred to directly measure the deformation of the vibrating mass of the transducer by gluing a piezoelectric patch on it. A resistor with a small value is then connected in parallel, so that the output voltage of the patch is directly proportional to the vibration speed of the PT corresponding to $u(t)$ in the equation (7). This way, a current sensing circuit is not necessary. The sensor has been calibrated by comparing the signal's output with the vibration amplitude measurement from a laser interferometer. The sensor's voltage shows a good linearity with the vibration velocity $u(t)$.

The frequency response of the transducer is given figure 13. This response is highly non linear, and the resonance frequency shifts due to variations of the stiffness with the vibration amplitude; this non linear behavior justifies the necessity to track the resonant frequency, while controlling the vibration speed.

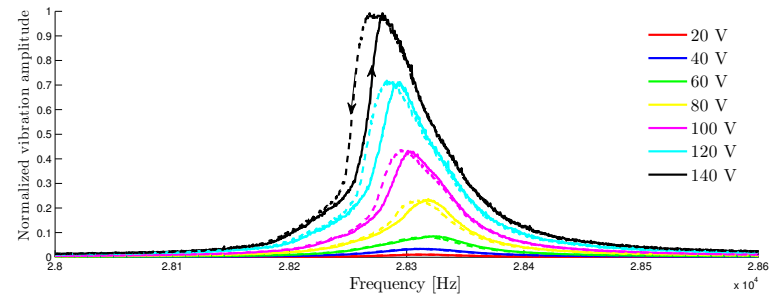

Fig. 13. Measured vibration amplitude curves for different driving signals according to up and down (plain line) frequency (dashed line)
Figure 14 shows the behavior of the controller when reaching the resonance frequency.
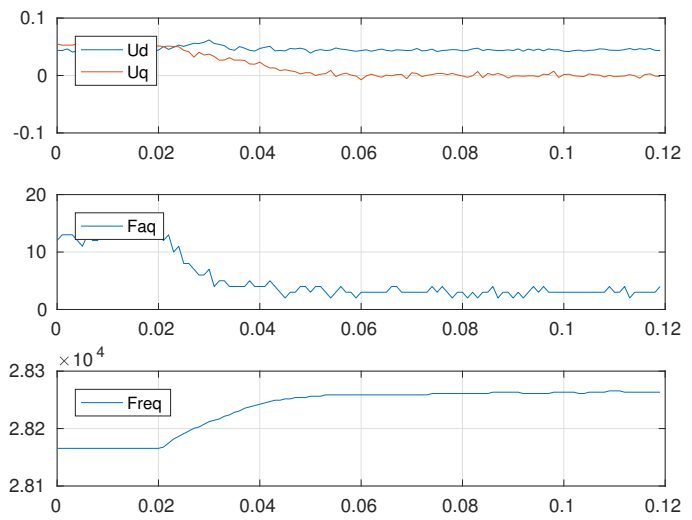

Fig. 14. Resonance Frequency Tracking

At $t<0.02 \mathrm{~s}$ the frequency controller is not activated, and the PT operates below the resonance, as a consequence $U_{d} \neq$ 0 . At $t=0.02 \mathrm{~s}$, the frequency tracker is switched on: the frequency increases in order to reach the value where $U_{d}=$ 0 is verified. Since the PT operates closer to the resonance, the supply voltage $V_{q}$ decreases in order to maintain $U_{q}$ to its reference setpoint. To reach the final value, the controller requires only $0.020 \mathrm{~s}$. Figure 15 presents the transient response of the PT when a step variation of $U_{q}$ is applied. The two controllers on each axis were activated.
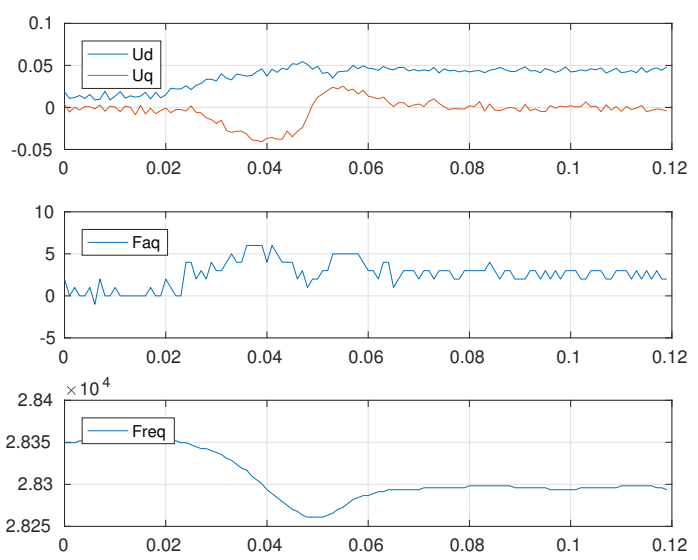

Fig. 15. Response of the PT to step variation of the reference

During this test, due to the non-linear amplitude vs frequency response presented in figure 13, the resonance frequency of the system shifts by $50 \mathrm{~Hz}$, which is large compared with the bandwidth of the system (aproximately $100 \mathrm{~Hz}$ ). Figure 15 clearly shows that first, the voltage $U_{q}$ increases to reach the reference setpoint. Doing so, the PT shifts away from its resonance and $U_{q}$ decreases. Consequently, $\delta \omega$ decreases by the action of the corrector in axis $d$. At $t=0.05 \mathrm{~s}$, the value of $U_{d}$ is stabilized, but $U_{q}$ is positive, meaning that the PT now 
operates below its resonance. Therefore, the frequency tracker increases $\omega$ to reach the resonance frequency, as previously shown in figure 14.

\section{Application to the synchronization of two Piezoelectric Transducers}

In some applications, two or more actuators are coupled to excite several modes. For instance, Traveling Wave Ultrasonic Motors (TWUM) use two vibration modes in quadrature to propel by friction a rotor pressed on it. A pure progressive wave is then obtained when the two modes vibrate with a temporal phase shift of $90 \mathrm{deg}$ [21], [22]. In some cases, the two modes are of different nature [23], and therefore do not necessarily have the same resonant frequency. In [24], the authors use a vibrating plate to manipulate cells according to patterns produced by the vibration of two orthogonal modes. All these examples have in common the necessity to control the phase and amplitude of the two modes which, in practice, do not have the same resonance frequency. For the best performance, precise adjustment of the amplitude, phase and frequency of the supply voltages is required [25]. In this paper, we apply the Vector Control Method on a 2-fingers tactile stimulator. By combining two vibration modes with particular amplitudes, it is possible to produce tactile stimuli on two fingers independently [26]. With the proposed closed loop control, we can control the vibration amplitude under two fingers located anywhere on the plate.

The device is a $140 \times 140 \times 4 \mathrm{~mm}^{3}$ plate made with aluminum. Two pairs of piezoelectric actuators glued on the plate excite two bending modes, one along axis $x$ the other along axis $y$, which are denoted by Mode $\mathrm{X}$ and Mode $\mathrm{Y}$ hereafter. The two deformation mode shapes $\Phi_{X}(x, y)$ and $\Phi_{Y}(x, y)$ are depicted figure 16 . Two more patches are glued in order to measure the vibration velocity of each mode by the direct piezoelectric effect.

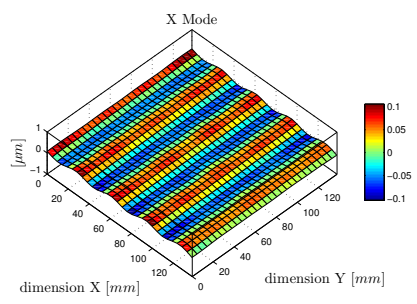

(a)

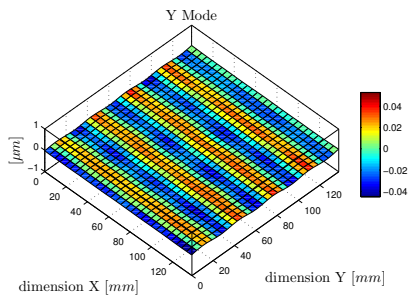

(b)
Fig. 16. Measured deformation mode shapes at resonance frequency of Mode $X$ at $33011 \mathrm{~Hz}$ and Mode $\mathrm{Y}$ at $33065 \mathrm{~Hz}$

When the two modes are vibrating at the same frequency, the vibration amplitude depends on the position on the plate, and we write:

$$
w(x, y, t)=\Re\left(\underline{W}_{X} \Phi_{X}+\underline{W}_{Y} \Phi_{Y}\right) e^{j \omega t}
$$

Where $W_{X}$ and $W_{Y}$ are the vibration amplitudes of the two modes. Hence, by setting the two modes with different amplitude, several vibration patterns can be obtained.However, the two modes are not at the same frequency, and their frequency response depicted figure 17 shows that mode $\mathrm{X}$ is at $33011 \mathrm{~Hz}$, while mode $\mathrm{Y}$ is at $33065 \mathrm{~Hz}$. The difference is small (less than 0.02\%); however, we can see that the resonance of one mode falls outside the bandwidth of the other mode, and setting the frequency at one mode's resonance would lead to high voltage requirement for the other. Operating in the middle of the two modes is a trade-off, which would lead to control the amplitude and the phase directly with the voltage of each mode. In practice however, this condition is difficult to attain, because the resonance frequencies shift due to temperature rise and/or the environment. Therefore in openloop, synchronizing the two modes needs a fine tuning of the voltages, in amplitude and phase [25].

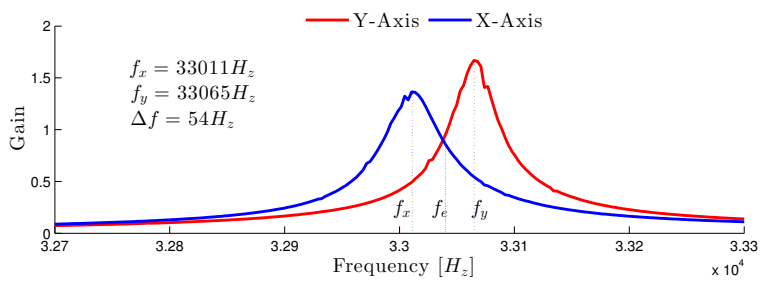

Fig. 17. Frequency response of the tactile plate

In the rotating reference frame, synchronizing the two modes is achieved by setting $U_{d X r e f}=U_{d Y r e f}$, and $U_{q X r e f}=U_{q Y r e f}$. We then implemented two VCM, one for each mode, leading to four control loops, working at a constant frequency chosen approximately in the middle of the two modes. Hence, the frequency tracking is not activated. The response time of the control is about $20 \mathrm{~ms}$ as can be seen in figure (18).

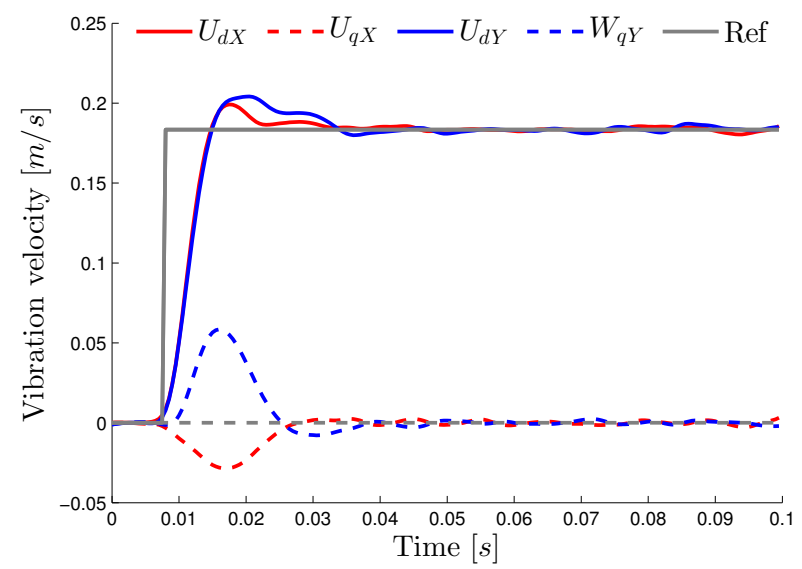

Fig. 18. Experimental measurement of the vibration amplitude control of mode $X$ and mode $Y$ in a rotating frame.

The result of this superimposition is given in figure (19). Compared with the measurements of the figure 16 the nodal line (where $w(x, t)=0$ ) are straight lines oriented at $45^{0}$, demonstrating the good synchronization of the two modes.

\section{CONCLUSION}

This paper presents the implementation of the Vector Control Method on Ultrasonic Transducers. We show that the 


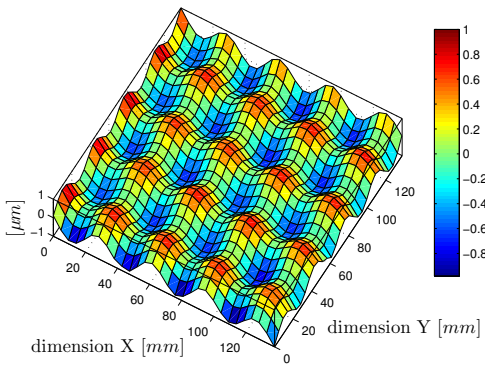

Fig. 19. Experimental measurement of superimposition of two vibration modes $\mathrm{X}$ and $\mathrm{Y}$ with $W_{X}=W_{Y}$.

VCM of a PT is very similar to the VCM of an Electromagnetic machine. However, since the couplings between the axis $d$ and $q$ strongly depend on the frequency of the supply voltage, we show how they can be either compensated in a fixed frequency control, or inverted in order to achieve a resonant frequency tracking. The originality of the proposed method is that the vibration amplitude controller and the frequency controller are identical, with the same parameters. The real-time implementation achieved with a low-cost DSP is based on a DDS for the matrices inversion. Good performances are obtained with simple PI controllers, since in steady state, the variables in the rotating reference frame are constant. The performances of the control are measured on a non-linear PT, and on a 2 DoF Tactile display where the synchronization of two vibration modes is critical.

Compared with other closed loop controls, the advantage of the VCM are the synchronization of several PTs, the possibility to operate the frequency tracking in parallel with the vibration amplitude controller, and the generality of the approach which can be applied to several types of PT's. Future work will focus on the extension of the proposed method to more than 2 modes.

\section{REFERENCES}

[1] F. Wang, H. Zhang, C. Liang, Y. Tian, X. Zhao, and D. Zhang, "Design of high-frequency ultrasonic transducers with flexure decoupling flanges

[2] X. Liu, A. I. Colli-Menchi, J. Gilbert, D. A. Friedrichs, K. Malang, and E. Sánchez-Sinencio, "An automatic resonance tracking scheme with maximum power transfer for piezoelectric transducers," IEEE Transactions on Industrial Electronics, vol. 62, no. 11, pp. 7136-7145, Nov 2015

[3] T. J. Mason, "Ultrasonic cleaning: An historical perspective," Ultrasonics Sonochemistry, vol. 29, pp. 519 - 523, 2016.

[4] G. P. L. Thomas, M. A. B. Andrade, J. C. Adamowski, and E. C. N. Silva, "Development of an acoustic levitation linear transportation system based on a ring-type structure," IEEE Transactions on Ultrasonics, Ferroelectrics, and Frequency Control, vol. 64, no. 5, pp. 839-846, 2017.

[5] C. Zhao, Ultrasonic motors: technologies and applications. Springer Science \& Business Media, 2011.

[6] E. Vezzoli, W. B. Messaoud, M. Amberg, F. Giraud, B. LemaireSemail, and M.-A. Bueno, "Physical and perceptual independence of ultrasonic vibration and electrovibration for friction modulation," IEEE transactions on haptics, vol. 8, no. 2, pp. 235-239, 2015.

[7] F. Bai, K.-A. Saalbach, J. Twiefel, and J. Wallaschek, "Effect of different standoff distance and driving current on transducer during ultrasonic cavitation peening," Sensors and Actuators A: Physical, vol. 261, pp. $274-279,2017$. for thermosonic bonding," IEEE Transactions on Industrial Electronics, vol. 63, no. 4, pp. 2304-2312, April 2016.

[8] F. Lim, M. Cartmell, A. Cardoni, and M. Lucas, "A preliminary investigation into optimising the response of vibrating systems used for ultrasonic cutting," Journal of Sound and Vibration, vol. 272, no. 3, pp. 1047 - 1069, 2004.

[9] S. Hayashi, "On the tracking of resonance and antiresonance of a piezoelectric resonator," IEEE Transactions on Ultrasonics, Ferroelectrics, and Frequency Control, vol. 38, no. 3, pp. 231-236, May 1991.

[10] Y. Kuang, Y. Jin, S. Cochran, and Z. Huang, "Resonance tracking and vibration stablilization for high power ultrasonic transducers," Ultrasonics, vol. 54, no. 1, pp. 187 - 194, 2014.

[11] L. C. Cheng and C. L. Chen, "A resonant frequency tracking circuit for a low-coupling-coefficient piezoelectric transducer," in 2014 IEEE Conference on Energy Conversion (CENCON), Oct 2014, pp. 377-382.

[12] L. Gui, R. Xiao, X. Liao, Y. Kuang, M. Sadiq, C. Zeng, S. Cochran, and Z. Huang, "Automatic frequency tracking system for needle actuating device," in 2014 IEEE International Ultrasonics Symposium, Sept 2014, pp. $815-818$.

[13] W. Ben Messaoud, M.-A. Bueno, and B. Lemaire-Semail, Textile Fabrics' Texture: From Multi-level Feature Extraction to Tactile Simulation. Cham: Springer International Publishing, 2016, pp. 294-303.

[14] A. Mathieson, R. Wallace, R. Cleary, L. Li, H. Simpson, and M. Lucas, "Ultrasonic needles for bone biopsy," IEEE Transactions on Ultrasonics, Ferroelectrics, and Frequency Control, vol. 64, no. 2, pp. 433-440, 2017.

[15] S. Shinnaka, "New sensorless vector control using minimum-order flux state observer in a stationary reference frame for permanent-magnet synchronous motors," IEEE Transactions on Industrial Electronics, vol. 53, no. 2, pp. 388-398, April 2006.

[16] W. B. Messaoud, F. Giraud, B. Lemaire-Semail, M. Amberg, and M. A. Bueno, "Amplitude control of an ultrasonic vibration for a tactile stimulator," IEEE/ASME Transactions on Mechatronics, vol. 21, no. 3, pp. 1692-1701, June 2016.

[17] S. Ghenna, F. Giraud, C. Giraud-Audine, M. Amberg, and B. LemaireSemail, "Modelling and control of a travelling wave in a finite beam, using multi-modal approach and vector control method," in 2015 Joint Conference of the IEEE International Frequency Control Symposium the European Frequency and Time Forum, April 2015, pp. 509-514.

[18] A. L. Allegre, A. Bouscayrol, P. Delarue, P. Barrade, E. Chattot, and S. El-Fassi, "Energy storage system with supercapacitor for an innovative subway," IEEE Transactions on Industrial Electronics, vol. 57, no. 12, pp. 4001-4012, Dec 2010.

[19] F. Giraud, M. Amberg, and B. Lemaire-Semail, "Design and control of a haptic knob," Sensors and Actuators A: Physical, vol. 196, pp. 78 $85,2013$.

[20] D. Yamaguchi, Y. Ochiai, T. Hoshi, J. Rekimoto, and M. Takasaki, Driving System of Diminished Haptics: Transformation of Real-World Textures. Tokyo: Springer Japan, 2015, pp. 63-68.

[21] W. Shi, H. Zhao, J. Ma, and Y. Yao, "An optimum-frequency tracking scheme for ultrasonic motor," IEEE Transactions on Industrial Electronics, vol. 64, no. 6, pp. 4413-4422, June 2017.

[22] T. Mashimo, "Micro ultrasonic motor using a cube with a side length of 0.5mm," IEEE/ASME Transactions on Mechatronics, vol. 21, no. 2, pp. 1189-1192, April 2016.

[23] X. Zhou, W. Chen, and J. Liu, "Novel 2-dof planar ultrasonic motor with characteristic of variable mode excitation," IEEE Transactions on Industrial Electronics, vol. 63, no. 11, pp. 6941-6948, Nov 2016.

[24] S. Oberti, A. Neild, and J. Dual, "Manipulation of micrometer sized particles within a micromachined fluidic device to form two-dimensional patterns using ultrasound," The Journal of the Acoustical Society of America, vol. 121, no. 2, pp. 778-785, 2007.

[25] A. Minikes, R. Gabay, I. Bucher, and M. Feldman, "On the sensing and tuning of progressive structural vibration waves," IEEE Transactions on Ultrasonics, Ferroelectrics, and Frequency Control, vol. 52, no. 9, pp. 1565-1576, Sept 2005.

[26] S. Ghenna, C. Giraud-Audine, F. Giraud, M. Amberg, and B. LemaireSemail, "Control and evaluation of a 2-d multimodal controlled-friction display," in IEEE World Haptics, Fürstenfeldbruck (Munich), Germany, Jun. 2017 